\title{
Colonic graft-vs.-host disease in autologous versus allogeneic transplant patients: earlier onset, more apoptosis, and lack of regulatory T-cell attenuation
}

\author{
Christopher P. Hartley ${ }^{1} \cdot$ Luis F. Carrillo-Polanco ${ }^{1} \cdot$ Daniel J. Rowan $^{1} \cdot$ Catherine E. Hagen $^{1}$
}

Received: 22 February 2018 / Revised: 25 April 2018 / Accepted: 25 April 2018 / Published online: 13 June 2018

(c) United States \& Canadian Academy of Pathology 2018

\begin{abstract}
Histologic characterization of graft-vs.-host disease in autologous stem cell transplant patients has been limited. The aims of this study were to characterize colonic graft-vs.-host disease in autologous stem cell transplant patients and compare to a control group of allogeneic stem cell transplant patients, to determine whether graft-vs.-host disease can be diagnosed $<21$ days post transplantation in autologous stem cell transplant recipients, and to quantify colonic T-cell populations in autologous stem cell transplant patients. Colonic biopsies taken to evaluate for graft-vs.-host disease in both allogenic and autologous stem cell transplant patients were reviewed for the maximum number of apoptotic bodies per 10 contiguous crypts. Immunohistochemical stains for CD4, CD8, and FoxP3 were performed. Clinical information was collected from chart review. The study group consisted of 122 colonic biopsies from 84 patients. Sixteen patients underwent autologous stem cell transplant and 68 allogeneic stem cell transplant. Autologous stem cell transplant patients underwent biopsy significantly earlier compared with allogeneic stem cell transplant patients (median 20 vs. 87 days, $p=0.0002$ ), had significantly higher apoptotic counts compared with matched-related donor patients (7.5 vs. 3.9, $p=0.03$ ), and had higher FoxP3-positive lamina propria lymphocytes counts compared to allogeneic stem cell transplant patients (9.2 vs. 5.3, $p=0.03$ ). In patients undergoing biopsy $<21$ days post transplantation, allogeneic stem cell transplant patients showed less CD8-positive lamina propria lymphocytes and a trend of less FoxP3- and CD4-positive lamina propria lymphocytes compared with autologous stem cell transplant patients. Autologous stem cell transplant patients have more prominent crypt apoptosis compared with allogenic stem cell transplant patients and do not have numerically decreased FoxP3-positive lamina propria lymphocytes. Presence of robust T-cell populations in the early period following transplantation suggest that the 21-day cutoff for diagnosis of graft-vs.-host disease is not applicable to autologous stem cell transplant patients.
\end{abstract}

\section{Introduction}

Graft-vs.-host disease is a well-known complication of allogeneic stem cell transplantation but also occurs in a subset of autologous stem cell transplant patients. Similar to graft-vs.-host disease occurring in the allogeneic stem cell

Electronic supplementary material The online version of this article (https://doi.org/10.1038/s41379-018-0078-7) contains supplementary material, which is available to authorized users.

Catherine E. Hagen

cahagen@mcw.edu

1 Department of Pathology, Medical College of Wisconsin, Milwaukee, WI, USA transplant population, autologous graft-vs.-host disease occurs in the skin, gastrointestinal tract, and liver. The reported incidence in the gastrointestinal tract ranges from $4 \%$ to $13 \%$ with a reported incidence at our own institution of 4\% [1-5]. Although graft-vs.-host disease occurring spontaneously in autologous stem cell transplant patients seems counterintuitive due to lack of genetic disparity, it is thought to arise secondary to failure of self-tolerance. Regulatory $\mathrm{T}$ cells are a major mediator of self-tolerance and it has been postulated that chemotherapy regimens may diminish regulatory T-cell populations, ultimately allowing the development of autologous graft-vs.-host disease [5, 6].

Autologous stem cell transplant patients develop symptoms of gastrointestinal graft-vs.-host disease on average 15 days post transplant, with some developing symptoms as early as a few days following stem cell transplant [2]. 
Colonoscopy with diagnostic biopsy has been performed as early as 13 days post transplant in previous reports [5, 6]. However, it is a commonly held belief that colonic graft-vs.host disease cannot be confidently diagnosed on biopsy < 21 days after stem cell transplant because the cytotoxic effects of conditioning chemotherapy and total body irradiation cause histologic changes indistinguishable from graft-vs.-host disease [7, 8].

Histologic characterization of autologous graft-vs.-host disease in the gastrointestinal tract has been limited. Gastric autologous graft-vs.-host disease has been characterized by a lymphocytic gastritis pattern as well as gastric pit apoptosis [3]. Cases of autologous graft-vs.-host disease in the colon have been reported to have crypt apoptosis, crypt dropout, and ulceration similar to allogeneic graft-vs.-host disease $[2,5,6]$. Formal histologic comparison of autologous and allogeneic gastrointestinal graft-vs.-host disease cases has not been performed.

The aims of this study were to histologically and clinically characterize gastrointestinal graft-vs.-host disease in autologous stem cell transplant patients and compare to a control group of allogeneic stem cell transplant patients, to determine whether gastrointestinal graft-vs.-host disease can be reliably diagnosed $<21$ days post transplantation in autologous stem cell transplant recipients, and to quantify $\mathrm{T}$-cell populations, including regulatory $\mathrm{T}$ cells, within the colonic mucosa of autologous stem cell transplant patients.

\section{Methods}

This study was approved by the Institutional Review Board. The pathology database at our institution was retrospectively searched for colon biopsies taken to rule out graft-vs.-host disease in both allogeneic and autologous stem cell transplant patients from 2008 to 2014. Biopsies with histologic evidence of cytomegalovirus (CMV) colitis and cases from solid organ transplant patients or asymptomatic patients were excluded.

Original hematoxylin and eosin-stained slides were blindly reviewed by one pathologist with experience in gastrointestinal pathology (CEH) for the maximum number of apoptotic bodies per 10 contiguous crypts. Cases were also evaluated for evidence of crypt dropout or ulceration and graded according to the Lerner criteria [9]. In patients with multiple biopsy specimens for an endoscopy procedure, the highest apoptotic count and highest Lerner grade were used for analysis. The number of serial sections examined and number of biopsy fragments per endoscopy were recorded. If available, corresponding upper gastrointestinal tract biopsies were also reviewed and graded according to the Lerner criteria.
A tissue microarray was subsequently constructed using 14 biopsies from 14 of the autologous stem cell transplant patients and 56 biopsies from 50 of the allogeneic stem cell transplant patients. Seventeen consecutive normal colon biopsies from 16 patients without a history of stem cell transplant were also included as a control group. Two or three $1 \mathrm{~mm}$ cores from each biopsy specimen were used to create the tissue microarray. Immunohistochemistry for CD4 (mouse monoclonal, clone 4B12, catalog no. IR649, Agilent-Dako, Santa Clara, CA, USA), CD8 (mouse monoclonal, clone C8/144B, catalog number IR623, Agilent-Dako), and FoxP3 (mouse monoclonal, clone 236A/E7, catalog number ab20034, dilution 1:200, Abcam, Cambridge, MA, USA) was performed on sections from the tissue microarray to evaluate for helper $\mathrm{T}$ cells, cytotoxic $\mathrm{T}$ cells, and regulatory $\mathrm{T}$ cells, respectively. The number of positively staining intraepithelial lymphocytes in both the crypt and surface epithelium and lamina propria lymphocytes was counted manually for each of the three immunohistochemical markers and averaged per high powered field (field diameter $0.50 \mathrm{~mm}$ ) for each specimen. Areas with mucosal lymphoid aggregates were excluded from evaluation.

Clinicopathologic information including underlying disease, patient symptoms, time from transplant to biopsy, type of transplant, conditioning chemotherapy regimens, treatment information, symptom resolution, and evidence of graft-vs.-host disease at extracolonic sites was collected through chart review. In patients undergoing biopsy< 21 days post transplantation, absolute neutrophil count at time of biopsy was recorded. Information collected for type of transplantation included autologous vs. allogeneic, peripheral blood stem cell transplantation vs. bone marrow transplantation vs. cord blood transplantation, and for patients undergoing allogeneic stem cell transplant, matched-related donor vs. matched-unrelated donor. Resolution of symptoms was considered resolution of symptoms within 1 month of treatment.

$T$-test, Mann-Whitney $U$-test, or one-way analysis of variance (ANOVA) was used to compare continuous variables between groups and chi-square test or Fisher's exact test was used to compare categorical variables by use of the Prism statistical program (GraphPad, 2015, San Diego, CA, USA).

\section{Results}

\section{Patient population}

The study group consisted of 122 colonic biopsies from 84 patients and 97 endoscopy procedures. Sixteen patients had undergone autologous stem cell transplant and 68 
patients had undergone allogeneic stem cell transplant. The mean age of the autologous stem cell transplant group was 59 years and the mean age of the allogeneic stem cell transplant group was 53 years $(p=0.07)$. Seven patients in the autologous stem cell transplant group were male and 9 female (M:F 1:1.3), and 30 allogeneic stem cell transplant patients were male and 38 female (M:F 1:1.3). One autologous stem cell transplant patient had two sets of biopsies included in the study, 10 allogeneic stem cell transplant patients had 2 sets of biopsies, and 1 allogeneic stem cell transplant patient had 3 sets of biopsies. Clinicopathologic data for patients with repeat endoscopies are provided in Supplemental Table 1. In the autologous group, the original indication for transplantation was multiple myeloma in 13 patients and non-Hodgkin lymphoma in 3 patients. Indications for transplantation in the allogeneic stem cell transplant group were as follows: acute myelogenous leukemia $(n=30)$, non-Hodgkin lymphoma $(n=16)$, multiple myeloma $(n=9)$, acute lymphoblastic leukemia $(n=6)$, myelofibrosis $(n=2)$, myelodysplastic syndrome $(n=2)$, classical Hodgkin lymphoma $(n=1)$, acute promyelocytic leukemia $(n=1)$, and aplastic anemia $(n=1)$.

Conditioning chemotherapy regimens for allogeneic stem cell transplant patients included: busulfan/cyclophosphamide $(\mathrm{Bu} / \mathrm{Cy})(n=10)$, Fludarabine $(\mathrm{Flu})$ and total body irradiation $(n=10), \mathrm{Bu} / \mathrm{Flu}(n=9), \mathrm{Bu} / \mathrm{Flu}$ reduced intensity $(n=6)$, "nonmyeloablative conditioning" without further specification $(n=6)$, Cy/total body irradiation $(n=$ 6), total body irradiation $200 \mathrm{cGy}(n=3), \mathrm{Bu} / \mathrm{Cy}+$ antithymocyte globulin (ATG) $(n=2), \quad$ Flu/Cy/Rituxan $(n=2), \mathrm{Bu} / \mathrm{Cy}+$ etoposide $(n=1), \mathrm{Bu} / \mathrm{Flu} / \mathrm{ATG}(n=1)$, $\mathrm{Bu} / \mathrm{Flu} / \mathrm{ATG} /$ total body irradiation $(n=1), \mathrm{Bu} / \mathrm{Flu} / \mathrm{Cy}(n=$ 1), Cy/ATG/ total body irradiation $(n=1)$, carmustine/ etoposide/cytarabine/melphalan (BEAM) $(n=1), \mathrm{Flu} / \mathrm{Cy}$ $(n=1), \mathrm{Flu} / \mathrm{Cy} / \mathrm{ATG}(n=1), \mathrm{Flu} / \mathrm{Cy} /$ total body irradiation $(n=1)$, Flu/melphalan (mel) $(n=1)$, mel/thiotepa (thio)/ Flu/ATG $(n=1)$, and thio/flu/ATG/ total body irradiation $(n=1)$. Conditioning chemotherapy regimens for autologous stem cell transplant patients included melphalan in 11 patients and BEAM in 3 patients. Clinical data regarding conditioning chemotherapy was not available for two allogeneic stem cell transplant patients and two autologous stem cell transplant patients.

All patients undergoing autologous stem cell transplant received peripheral blood stem cell transplantation. Within the allogeneic stem cell transplant group, 48 patients (71\%) received peripheral blood stem cell transplant, 17 (25\%) bone marrow transplantation, and $3(4 \%)$ cord blood transplantation. Thirty-six (53\%) allogeneic stem cell transplant patients received stem cell transplant from a matched-unrelated donor and $32(47 \%)$ from a matchedrelated donor.

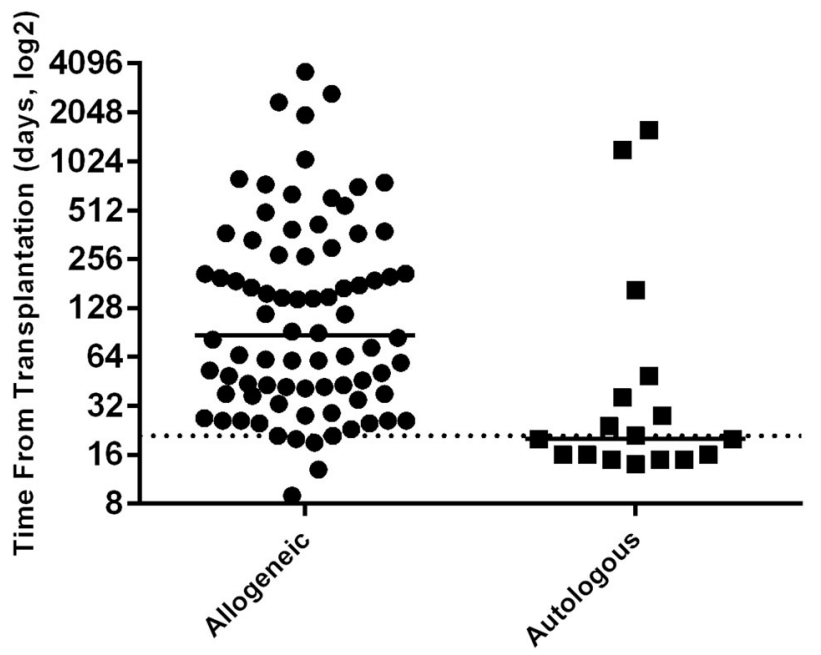

Fig. 1 Number of days post transplant on which biopsy was performed in allogeneic vs. autologous stem cell transplant patients. Dotted line represents 21 days post transplant and solid lines represent median for each group

\section{Timing of biopsy}

A total of 13 patients had biopsy $<21$ days post transplant (9 autologous and 4 allogeneic). All nine autologous stem cell transplant patients undergoing biopsy $<21$ days post transplantation had a documented absolute neutrophil count $\geq 500 / \mu \mathrm{L}$ for at least 3 consecutive days at the time of biopsy. Only one of the allogenic stem cell transplant patients undergoing biopsy $<21$ days post transplantation had a documented absolute neutrophil count $\geq 500 / \mu \mathrm{L}$ for at least 3 consecutive days at the time of biopsy. Autologous stem cell transplant patients underwent biopsy significantly earlier following transplantation compared with allogeneic stem cell transplant patients (median 20 vs. 87 days, $p=0.0002$ by Mann-Whitney test) and were also significantly more likely to undergo biopsy $<21$ days post transplant $(56 \%$ vs. $6 \%, p<0.0001)$ (Fig. 1).

\section{Histologic findings}

The number of biopsy fragments per endoscopy was similar in autologous stem cell transplant (mean 11, range 5-16) and allogeneic stem cell transplant patients (mean 11, range $3-26, p=0.66)$. The number of serial sections examined per biopsy in autologous (mean 10, range 6-12) and allogeneic stem cell transplant (mean 9, range $3-16, p=0.16$ ) patients was also statistically similar.

Within the autologous stem cell transplant group, seven biopsies had evidence of grade 1 graft-vs.-host disease, three grade 2, four grade 3, and three grade 4 (Fig. 2). Within the allogeneic stem cell transplant group, 8 patients had biopsies negative for graft-vs.-host disease, 49 had evidence of grade 1 graft-vs.-host disease, 7 grade 2, 6 
Fig. 2 a Histologic examples of colonic graft-vs.-host disease in autologous stem cell transplant patients. Crypt apoptosis without crypt dropout (grade 1). b Single crypt dropout (grade 2). c Contiguous crypt dropout (grade 3). d Diffuse crypt dropout with ulceration (grade 4)
Fig. 3 a Comparison of maximum apoptotic counts per 10 contiguous crypts among groups. Apoptotic counts in allogeneic (Allo), autologous (Auto), matched-related donor recipients (MRD), and matchedunrelated donor recipients (MUD), all cases included. b Apoptotic counts in Auto and Allo patients undergoing biopsy $<21$ days post transplant and $\geq 21$ days post transplant. c Apoptotic counts in Allo, Auto, MRD and MUD, cases negative for graft-vs.-host disease excluded

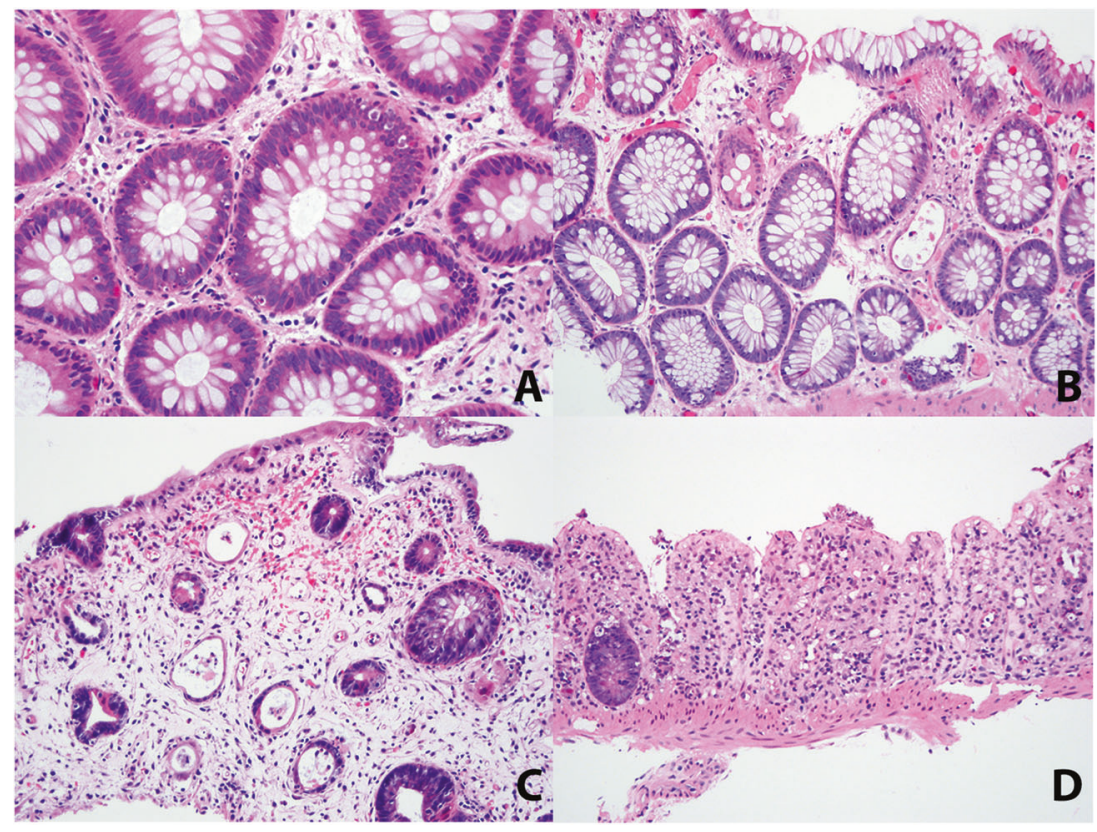

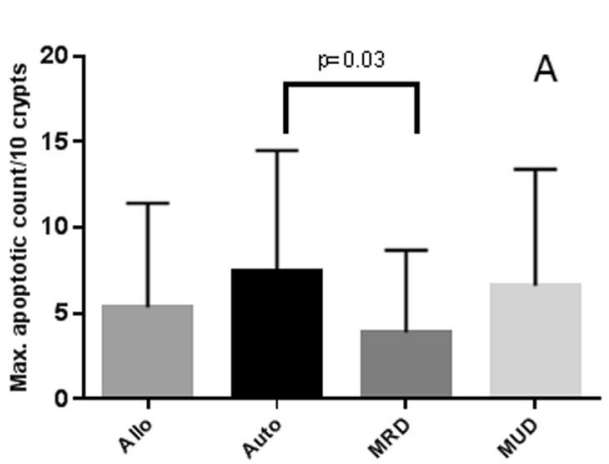

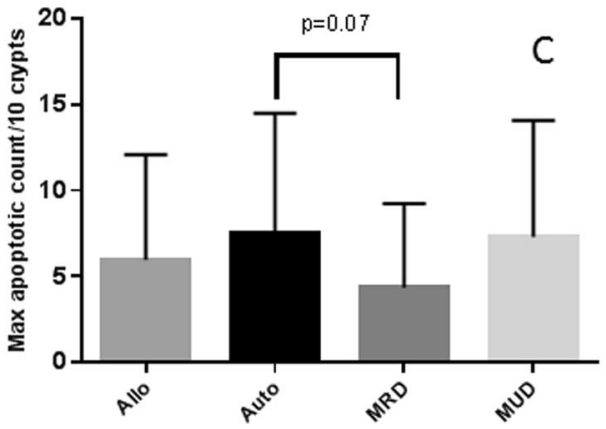

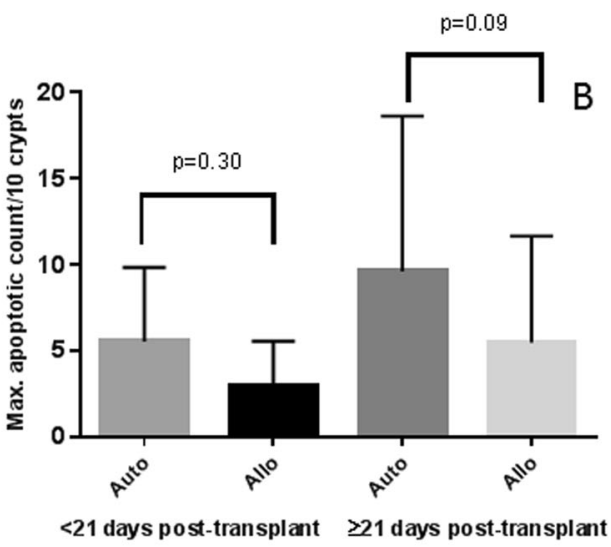

$B$ grade 3, and 10 grade 4. The distribution of histologic grades between autologous and allogeneic stem cell transplant was not significantly different $(p=0.11)$.

Autologous stem cell transplant patients exhibited higher maximum apoptotic counts per 10 contiguous crypts compared to allogeneic stem cell transplant patients, but this difference did not reach statistical significance ( 7.5 vs. 5.4 , $p=0.21)$. When the allogeneic stem cell transplant group was subdivided into matched-unrelated donor recipients and matched-related donor recipients, autologous stem cell transplant patients exhibited significantly higher apoptotic counts compared with matched-related donor patients (7.5 vs. 3.9, $p=0.03$ ) and similar apoptotic counts as matchedunrelated donor patients (7.5 vs. 6.6, $p=0.67$ ). When biopsies from allogeneic stem cell transplant patients that were negative for graft-vs.-host disease were excluded from 
Table 1 Comparison of FoxP3 ,$+ \mathrm{CD} 8+$, and CD4+

lymphocytes in autologous stem cell transplant patients, allogeneic stem cell transplant patients, and normal controls with all biopsies included

\begin{tabular}{lccclll}
\hline & Auto SCT & Allo SCT & Normal & $\begin{array}{l}\text { Auto vs. } \\
\text { Allo } \\
P \text {-value }\end{array}$ & $\begin{array}{l}\text { Auto vs. } \\
\text { normal }\end{array}$ & $\begin{array}{l}\text { Allo vs. } \\
\text { normal }\end{array}$ \\
\hline & Mean lymphocytes/HPF (range) & $6.3(1-14)$ & $\mathbf{0 . 0 3}$ & 0.22 & 0.55 \\
\hline $\begin{array}{l}\text { LPL FoxP3 } \\
+\end{array}$ & $9.2(1-24)$ & $5.3(0-22)$ & & & & 0.91 \\
LPL CD8+ & $30.3(4-78)$ & $23.0(0-101)$ & $29.5(12-77)$ & 0.27 & 0.32 \\
LPL CD4+ & $32.6(8-67)$ & $25.3(0-83)$ & $34.5(14-50)$ & 0.14 & 0.71 & $\mathbf{0 . 0 4}$ \\
IEL FoxP3+ & $0.19(0-1)$ & $0.06(0-1.3)$ & $0.04(0-0.5)$ & 0.07 & 0.12 & 0.69 \\
IEL CD8+ & $1.8(0-5)$ & $4.1(0-45)$ & $4.0(0-10)$ & 0.29 & $\mathbf{0 . 0 3}$ & 0.95 \\
IEL CD4+ & $0.24(0-1)$ & $0.25(0-2)$ & $0.04(0-0.5)$ & 0.91 & 0.06 & 0.07 \\
\hline
\end{tabular}

Allo, allogeneic; Auto, autologous; HPF, high-power field; IEL, intraepithelial lymphocyte; LPL, lamina propria lymphocytes

Bold values indicate statistically significant $P$-value

\begin{tabular}{llll}
\hline & $\begin{array}{l}\text { Allo SCT } \\
\text { Mean lymphocytes/ HPF (range) }\end{array}$ & $\begin{array}{l}\text { Auto vs. Allo } \\
P \text {-value }\end{array}$ & Allo vs. normal \\
\hline LPL FoxP3+ & $5.6(0-22)$ & 0.06 & 0.70 \\
LPL CD8+ & $22.6(0-95)$ & 0.21 & 0.26 \\
LPL CD4+ & $26.1(0-83)$ & 0.20 & 0.07 \\
\hline
\end{tabular}

Allo, allogeneic; Auto, autologous; LPL, lamina propria lymphocytes
Table 2 Comparison of FoxP3 ,$+ \mathrm{CD} 8+$, and CD4+ lymphocytes in autologous stem cell transplant patients, allogeneic stem cell transplant patients, and normal controls with biopsies negative for graftvs.-host disease excluded from allogeneic stem cell transplant group analysis, autologous stem cell transplant patients again exhibited nonsignificant higher apoptotic counts (7.5 vs. 6.0, $\mathrm{p}=0.38$ ). Compared to matched-unrelated donor patients, autologous stem cell transplant patients still exhibited similar apoptotic counts (7.5 vs. 7.3, $p=0.94)$, but compared with matched-related donor patients, the difference in apoptotic counts was no longer statistically significant (7.5 vs. $4.4, p=0.07$ ) (Fig. 3).

In patients undergoing biopsy $<21$ days post transplant, autologous stem cell transplant patients exhibited higher apoptotic counts compared to allogeneic stem cell transplant patients although not statistically significant (5.6 vs. 3.0, $p$ $=0.30$ ). In patients undergoing biopsy $\geq 21$ days post transplant, autologous stem cell transplant patients again exhibited a higher apoptotic count (9.6 vs. 5.5, $p=0.09$ ) (Fig. 3).

\section{Immunohistochemical results}

When all cases were included, autologous stem cell transplant patients had a similar number of FoxP3-, CD4-, and CD8-positive lamina propria lymphocytes compared with the normal colon control group. Autologous stem cell transplant patients had significantly higher numbers of FoxP3-positive lamina propria lymphocytes compared to allogeneic stem cell transplant patients (9.2 vs. 5.3, $p=$ 0.03 ), and allogeneic stem cell transplant patients had significantly less CD4-positive lamina propria lymphocytes compared to normal controls (25.3 vs. 34.5, $p=0.04)$.
Autologous stem cell transplant patients had significantly less CD8-positive intraepithelial lymphocytes compared to normal controls ( 1.8 vs. $4.0, p=0.03$ ). No other differences were observed between the three groups in terms of FoxP3-, CD4-, or CD8-positive intraepithelial lymphocytes (Table 1). When biopsies from allogeneic stem cell transplant patients that were negative for graft-vs.-host disease were excluded from analysis $(n=7)$, there was no longer any significant difference in number of FoxP3, CD4, or CD8 lamina propria lymphocytes between groups (Table 2).

In patients undergoing biopsy $<21$ days post transplantation, allogeneic stem cell transplant patients showed less CD8-positive lamina propria lymphocytes (3.2 vs. 34.2, $p=0.02$ ) and a trend of less FoxP3 (0.6 vs. 10.9, $p=0.056$ ) and CD4 (13.7 vs. 34.3, $p=0.08$ ) positive lamina propria lymphocytes compared with autologous stem cell transplant patients. In patients undergoing biopsy $\geq 21$ days post transplantation, there was no difference in number of FoxP3 (5.7 vs. 6.1, $p=0.86$ ), CD8 (24.5 vs. 23.4, $p=0.91$ ), or CD4 (26.2 vs. 29.5, $p=0.66)$ positive lamina propria lymphocytes in allogeneic vs. autologous stem cell transplant patients (Fig. 4).

\section{Subset analysis in multiple myeloma patients}

In patients with an underlying diagnosis of multiple myeloma there was no significant difference in apoptotic counts between patients who had undergone autologous stem cell transplant vs. allogeneic stem cell transplant (7.9 vs. 7.5, 
Fig. 4 a Comparison of colonic lamina propria T-cell populations among groups. Mean number of FoxP3-positive lamina propria lymphocytes (LPLs) in allogeneic (Allo) and autologous (Auto) stem cell transplant patients undergoing biopsy $<21$ days post transplant and $\geq 21$ days post transplant. b Mean number of CD8 positive LPLs in Allo and Auto stem cell transplant patients undergoing biopsy $<21$ days post transplant and $\geq 21$ days post transplant. c Mean number of CD4 positive LPLs in Allo and Auto stem cell transplant patients undergoing biopsy $<21$ days post transplant and $\geq 21$ days post transplant
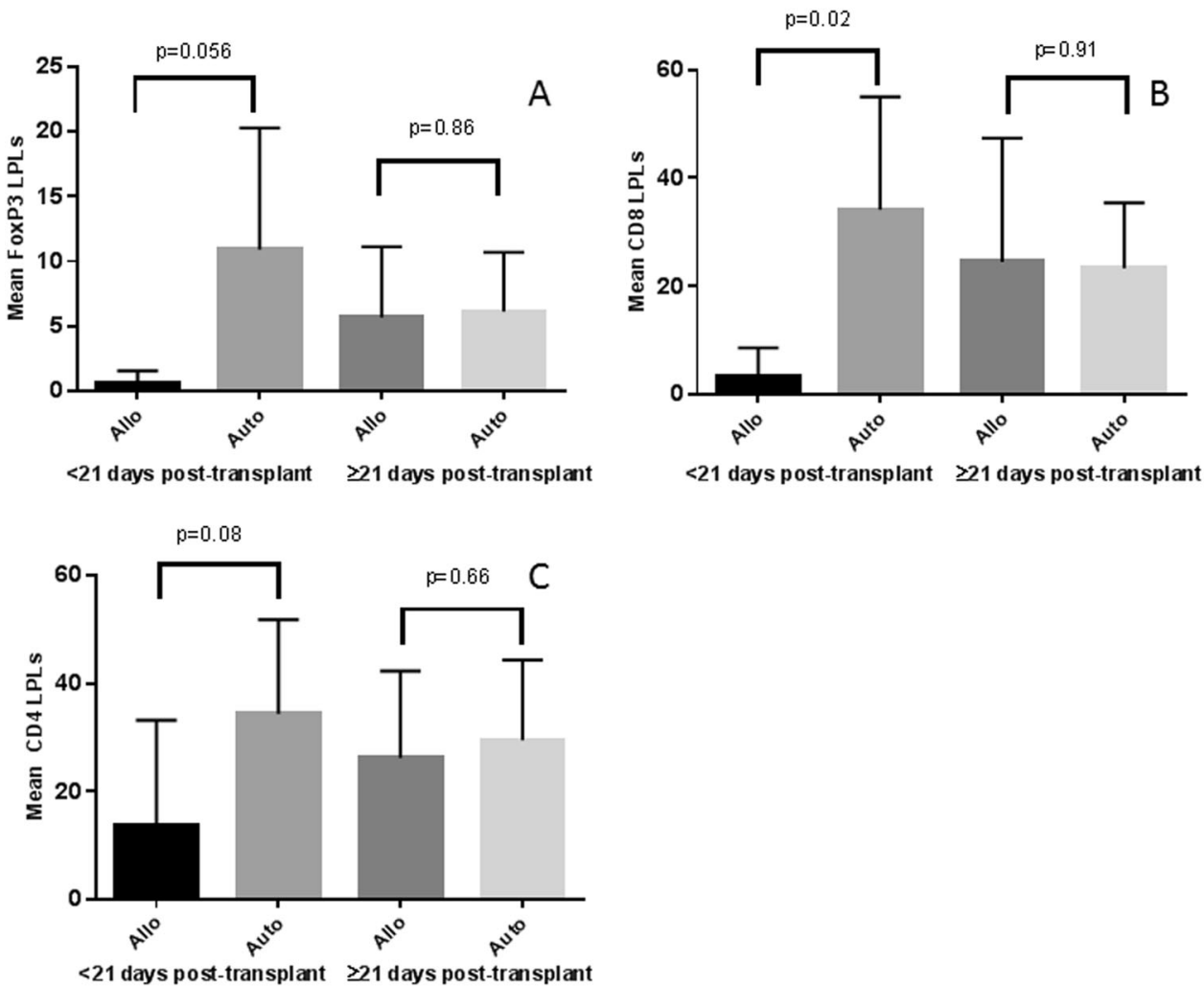

$p=0.91)$. When the allogenic stem cell transplant patients were subdivided into matched-related donor recipients and matched-unrelated donor recipients, there was still no statistical difference in apoptotic counts compared to autologous stem cell transplant patients $(p=0.90$ by one-way ANOVA).

Patients with multiple myeloma who underwent autologous stem cell transplant showed a nonsignificant trend of higher FoxP3-positive lamina propria lymphocytes (10.0 vs. $4.5, p=0.14)$, CD4-positive lamina propria lymphocytes (35.8 vs. $21.9, p=0.10$ ), and CD8-positive lamina propria lymphocytes (35.0 vs. $26.2, p=0.47)$ compared with multiple myeloma patients who underwent allogeneic stem cell transplant. Patients with multiple myeloma who underwent autologous stem cell transplant also showed a nonsignificant trend of higher FoxP3-positive intraepithelial lymphocytes $(0.2$ vs. $0, p=0.19)$ and CD4-positive intraepithelial lymphocytes $(0.2$ vs. $0.1, \mathrm{p}=0.26)$ compared with multiple myeloma patients who underwent allogeneic stem cell transplant. CD8-positive intraepithelial lymphocytes were similar in both autologous and allogeneic multiple myeloma patients (1.8 vs. $2.1, p=0.77$ ).

\section{Treatment and symptom resolution}

All autologous stem cell transplant patients received treatment for graft-vs.-host disease following biopsy, and all but one patient (94\%) had symptomatic response following treatment. The one patient that was initially refractory to treatment had improvement of diarrheal symptoms with addition of tincture of opium approximately 4 months after biopsy. Allogeneic stem cell transplant patients were treated following 69 of 80 (86\%) endoscopy procedures and 55 patients $(80 \%)$ had symptomatic response following treatment. Of the 11 untreated allogenic stem cell transplant patients, 3 had colonic biopsies negative for graft-vs.-host disease and the remaining 8 patients had only rare apoptosis ( $\leq 6$ apoptotic bodies per 10 contiguous crypts) on colonic biopsy.

\section{Evidence of infection and mycophenolate use}

Three autologous stem cell transplant patients had possible evidence of gastrointestinal infection at the time of biopsy (one adenovirus, one herpes simplex virus, and one Clostridium difficile colitis). CMV immunohistochemistry was performed on 16/17 autologous stem cell transplant colonic biopsies and all were negative. No autologous stem cell transplant patients were on mycophenolate at the time of biopsy.

Sixteen allogeneic stem cell transplant patients had possible evidence of gastrointestinal infection at the time of biopsy (14 C. difficile colitis, one Plesiomonas shigelloides, and one had coinfection of HHV-6 and adenovirus). CMV immunohistochemistry was performed on $66 / 80$ allogenic stem cell transplant colonic biopsies and all were negative. 
Twenty-eight allogeneic stem cell transplant patients were on mycophenolate at time of biopsy.

\section{Evidence of extracolonic graft-vs.-host disease}

Five autologous stem cell transplant patients had evidence of extracolonic graft-vs.-host disease (three skin and two upper gastrointestinal tract). Fifty-seven allogeneic stem cell transplant patients had evidence of extracolonic graft-vs.host disease, some of whom had evidence of graft-vs.-host disease at more than one site (40 skin, 22 liver, 3 upper gastrointestinal tract, 4 oral).

\section{Discussion}

Like allogeneic stem cell transplant patients, graft-vs.-host disease also occurs in a subset of autologous stem cell transplant patients. Thought to occur as a result of failure of self-tolerance, graft-vs.-host disease in autologous stem cell transplant patients commonly involves the gastrointestinal tract and has been reported to occur relatively early following transplantation $[2,5,6]$. To date, limited data has been published on the histologic features of graft-vs.-host disease occurring in autologous stem cell transplant patients as compared to allogenic stem cell transplant patients. Furthermore, early onset of graft-vs.-host disease in autologous stem cell transplant patients often warrants biopsy within the first few weeks following transplantation, challenging the long-held belief the graftvs.-host disease cannot be reliably diagnosed $<21$ post transplantation [7, 8].

We retrospectively reviewed a set of colon biopsies taken to evaluate for graft-vs.-host disease in 16 patients who underwent autologous stem cell transplant and compared to colon biopsies taken to evaluate for graft-vs.-host disease in 68 allogeneic stem cell transplant patients. Similar to a previous report, biopsies from autologous stem cell transplant patients showed the full spectrum of histologic features seen in allogeneic graft-vs.-host disease from rare apoptosis (grade 1) to extensive crypt dropout with ulceration (grade 4) [5]. Interestingly, none of the biopsies from autologous stem cell transplant patients were considered negative for graft-vs.-host disease (i.e. no apoptosis), whereas eight biopsies from the allogeneic stem cell transplant cohort were considered negative. This difference may be a result of varying thresholds of clinicians to perform biopsy in these two patient populations.

Biopsies from autologous stem cell transplant patients exhibited higher maximum apoptotic counts compared to allogeneic stem cell transplant patients, particularly in comparison to patients who underwent transplantation from a matched-related donor. Although the differing apoptotic counts may partly be attributed to differing conditioning chemotherapy regimens administered to autologous and allogeneic stem cell transplant patients, apoptotic counts were still higher in autologous stem cell transplant patients biopsied more than 21 days post transplantation. It therefore seems unlikely that differing conditioning regimens would be the only explanation for increased apoptosis in autologous stem cell transplant patients. It is possible that fundamentally graft-vs.-host disease occurring in autologous stem cell transplant patients is a more severe process, inducing more frequent apoptosis. However, when allogenic stem cell transplant patients with multiple myeloma were compared to autologous stem cell transplant patients with multiple myeloma, these two groups showed similar apoptotic counts arguing other factors than the type of transplantation may be responsible for differing apoptotic counts. Further studies with larger numbers of autologous stem cell transplant patients are necessary to support the apoptotic trends noted in this study.

Failure of self-tolerance is a major theory as to how graftvs.-host disease occurs in autologous stem cell transplant patients. Regulatory $\mathrm{T}$ cells are a major regulator of selftolerance and can be readily identified by nuclear positivity for FoxP3 immunohistochemistry. Limited studies have evaluated FoxP3-positive lymphocytes via immunohistochemistry in tissue biopsies from graft-vs.-host disease patients. A previous study has shown FoxP3-positive lymphocytes are not depleted in gastric biopsies from allogeneic stem cell transplant patients with evidence of graft-vs.-host disease [10]. In addition, Drobyski et al. [6] showed via flow cytometric analysis that numbers of FoxP3-positive lymphocytes were not decreased in grafts of autologous stem cell transplant patients who develop graft-vs.-host disease and Magenau et al. [11] showed that the frequency of regulatory $\mathrm{T}$ cells in the peripheral blood of autologous stem cell transplant patients was higher than that of allogeneic stem cell transplant patients who develop graft-vs.host disease. We found autologous stem cell transplant patients to have similar numbers of colonic FoxP3-positive lamina propria lymphocytes compared with normal controls and increased numbers compared with allogeneic stem cell transplant patients. Interestingly, when only patients with multiple myeloma were analyzed, autologous stem cell transplant patients again showed a trend of higher FoxP3positive lamina propria lymphocytes compared to allogenic stem cell transplant patients. Although our study did not address the functionality of regulatory $\mathrm{T}$ cells, our data does show that regulatory $\mathrm{T}$ cells are not numerically decreased in tissue biopsies from autologous stem cell transplant patients. In contrast to our findings, Batra et al. [12] noted a trend of less regulatory $\mathrm{T}$ cells via flow cytometric analysis in grafts of patients who develop autologous graft-vs.-host disease, but this finding did not reach statistical significance. 
Direct comparison between our study and this study is difficult given the different modalities used for identifying regulatory T cells. Only a minor subset of peripheral blood regulatory $\mathrm{T}$ cells have been shown to express gut-homing integrins and therefore measurement of peripheral blood regulatory $\mathrm{T}$ cells may not be an accurate representation of gastrointestinal tract regulatory $\mathrm{T}$ cells [13]. Future studies with concurrent immunohistochemical and flow cytometric analysis of regulatory $\mathrm{T}$ cells may provide further insight into the role of regulatory $\mathrm{T}$ cells in development of colonic autologous graft-vs.-host disease.

The majority (56\%) of autologous stem cell transplant patients in our study underwent biopsy $<21$ days post transplantation. The 21-day cutoff for diagnosis of graft-vs.host disease is based on data from studies on allogeneic bone marrow transplantation patients [7, 8]. It is well known that engraftment following bone marrow transplantation occurs later as compared to peripheral blood stem cell transplantation [14]. In addition, autologous stem cell transplant patients are a different patient population who undergo different conditioning regimens compared with allogeneic stem cell transplant patients. Our data also show that allogeneic stem cell transplant patients tend to have more of an attenuated T-cell population $<21$ days post transplantation, whereas autologous stem cell transplant patients do not. Therefore, it is feasible that diagnosis of graft-vs.-host disease could be made earlier in patients undergoing autologous peripheral blood stem cell transplantation. Future prospective studies are necessary to readdress this issue.

In summary, the results of our study show that the histologic features of gastrointestinal graft-vs.-host disease in autologous stem cell transplant patients are similar to those seen in allogeneic stem cell transplant patients, with the exception of more prominent crypt apoptosis. FoxP3positive lymphocytes are not numerically decreased in colonic biopsies from autologous stem cell transplant patients with evidence of graft-vs.-host disease, suggesting that numerical regulatory $\mathrm{T}$-cell attenuation is not a factor in the development of autologous graft-vs.-host disease. Finally, a large proportion of autologous stem cell transplant patients undergo biopsy for evaluation of graft-vs.-host disease $<21$ days post transplantation. Evidence of early engraftment and presence of robust T-cell populations in this early period following transplantation suggest that the 21-day cutoff for diagnosis of graft-vs.-host disease is not applicable to autologous stem cell transplant patients.
Acknowledgements We thank the staff of the pathology core laboratory for performing immunohistochemical stains for this project.

\section{Compliance with ethical standards}

Conflict of interest The authors declare that they have no conflict of interest.

\section{References}

1. Saunders MD, Shulman HM, Murakami CS, et al. Bile duct apoptosis and cholestasis resembling acute graft-versus-host disease after autologous hematopoietic cell transplantation. Am J Surg Pathol. 2000;24:1004-8.

2. Holmberg L, Kikuchi K, Gooley TA, et al. Gastrointestinal graftversus-host disease in recipients of autologous hematopoietic stem cells: incidence, risk factors, and outcome. Biol Blood Marrow Transplant. 2006;12:226-34.

3. Tzung SP, Hackman RC, Hockenbery DM, et al. Lymphocytic gastritis resembling graft-vs.-host disease following autologous hematopoietic cell transplantation. Biol Blood Marrow Transplant. 1998;4:43-48.

4. Hood AF, Vogelsang GB, Black LP, et al. Acute graft-vs-host disease. Development following autologous and syngeneic bone marrow transplantation. Arch Dermatol. 1987;123:745-50.

5. Cogbill CH, Drobyski WR, Komorowski RA. Gastrointestinal pathology of autologous graft-versus-host disease following hematopoietic stem cell transplantation: a clinicopathological study of 17 cases. Mod Pathol. 2011;24:117-25.

6. Drobyski WR, Hari P, Keever-Taylor C, et al. Severe autologous GVHD after hematopoietic progenitor cell transplantation for multiple myeloma. Bone Marrow Transplant. 2009;43:169-77.

7. Epstein RJ, McDonald GB, Sale GE, et al. The diagnostic accuracy of the rectal biopsy in acute graft-versus-host disease: a prospective study of thirteen patients. Gastroenterology. 1980;78:764-71.

8. Snover DC. Graft-versus-host disease of the gastrointestinal tract. Am J Surg Pathol. 1990;14:101-8.

9. Lerner KG, Kao GF, Storb R, et al. Histopathology of graft-vs.host reaction (GvHR) in human recipients of marrow from HL-Amatched sibling donors. Transplant Proc. 1974;6:367-71.

10. Lord JD, Hackman RC, Gooley TA, et al. Blood and gastric FOXP3 $+\mathrm{T}$ cells are not decreased in human gastric graft-versushost disease. Biol Blood Marrow Transplant. 2011;17:486-96.

11. Magenau JM, Qin X, Tawara I, et al. Frequency of CD4 $(+)$ CD25 (hi)FOXP3 $(+)$ regulatory $\mathrm{T}$ cells has diagnostic and prognostic value as a biomarker for acute graft-versus-host-disease. Biol Blood Marrow Transplant. 2010;16:907-14.

12. Batra A, Cottler-Fox M, Harville T, et al. Autologous graft versus host disease: an emerging complication in patients with multiple myeloma. Bone Marrow Res. 2014;2014:891427.

13. Hirahara K, Liu L, Clark RA, et al. The majority of human peripheral blood CD4 + CD25highFoxp3 + regulatory $\mathrm{T}$ cells bear functional skin-homing receptors. J Immunol. 2006;177:4488-94.

14. Holtick U, Albrecht M, Chemnitz JM, et al. Bone marrow versus peripheral blood allogeneic haematopoietic stem cell transplantation for haematological malignancies in adults. Cochrane Database Syst Rev 2014;4:CD010189. 